\title{
Industry updates from the field of stem cell research and regenerative medicine in July 2020
}

\author{
Dusko llic*,1(iD) \& Mirjana Liovic ${ }^{2}$ (iD) \\ ${ }^{1}$ Department of Women \& Children's Health, Stem Cell Laboratories, Guy's Assisted Conception Unit, Faculty of Life Sciences \& \\ Medicine, King's College London, London SE1 9RT, UK \\ ${ }^{2}$ Medical Center for Molecular Biology, Faculty of Medicine, University of Ljubljana, 1000 Ljubljana, Slovenia \\ *Author for correspondence: dusko.ilic@kcl.ac.uk
}

Latest developments in the field of stem cell research and regenerative medicine compiled from publicly available information and press releases from nonacademic institutions in July 2020.

First draft submitted: 29 August 2020; Accepted for publication: 5 October 2020; Published online: 4 November 2020

Keywords: industry $\bullet$ regenerative medicine $\bullet$ stem cells

\section{Business development}

Licensing agreement: Fate \& Baylor

Fate Therapeutics (CA, USA; https://fatetherapeutics.com), a clinical-stage biopharmaceutical company dedicated to the development of programmed cellular immunotherapies for cancer and immune disorders, has announced that the Company entered into an exclusive license agreement with Baylor College of Medicine (TX, USA; www.bcm.edu) covering alloimmune defense receptors (ADRs), a first-in-class approach that renders off-the-shelf allogeneic cell products resistant to host immune rejection [1]. Preclinical studies demonstrated that allogeneic cells engineered with a novel ADR are protected from both T- and NK-cell mediated rejection and provide proof-ofconcept that ADR-expressing allogeneic cell therapies can durably persist in immunocompetent recipients [2]. ADRs are synthetic receptors that selectively recognize cell surface receptors, such as 4-1BB, that are uniquely expressed on activated lymphocytes, which are responsible for host immune rejection. The preclinical findings show that the arming of allogeneic $\mathrm{T}$ cells with an ADR selectively eliminates alloreactive $\mathrm{T}$ and NK cells, while sparing resting lymphocytes. Importantly, in in vivo preclinical models of cancer, allogeneic $\mathrm{T}$ cells expressing both an ADR and a CD19-targeted chimeric antigen receptor (CAR) demonstrated increased expansion and persistence, resulting in sustained tumor eradication and a long-term survival benefit compared with conventional CD19-targeted CAR T cells.

Licensing agreement: QurAlis \& Eli Lilly

QurAlis Corporation (MA, USA; www.quralis.com), a biotech company focused on developing precision medicines for amyotrophic lateral sclerosis (ALS) and other neurologic diseases using patient-derived stem cell model, has announced an agreement to in-license pre-clinical compounds with disease modifying potential in ALS by preventing disease-induced neuronal excitotoxicity from Eli Lilly and Company (IN, USA; www.lilly.com) [3]. Financial terms were not disclosed. Excitotoxicity in ALS, a defining characteristic of the disease, is caused by the hyperactivity of neurons that leads to the internal buildup of toxic proteins and metabolic waste and the subsequent death of brain and spinal cord motor neurons. By aiming to prevent neuronal excitability with specifically targeted small molecule drugs, QurAlis' excitotoxicity research program intends to halt this process and prevent disease progression in those ALS patients, where this disease process plays a prominent role. 


\section{Launching new products, services... Merakris}

Merakris Therapeutics (NC, USA; www.merakris.com) has announced that the Centers for Medicare \& Medicaid Services has assigned a Healthcare Common Procedure Coding System product code Q4248 for its topical skin graft substitute, Dermacyte Matrix [4]. The new Healthcare Common Procedure Coding System code, effective 1 July 2020, enables physician office reimbursement under Medicare Part B. Dermacyte Matrix is a crosslinked amniotic membrane allograft designed for cutaneous wound applications, commonly used by outpatient surgical, podiatry and dermatology clinics. Dermacyte is available in dehydrated and hydrated configurations with 5 years of shelf stability at room temperature. Merakris has developed a novel purification system yielding two separate amniotic biomolecule fractions, one capable of promoting early-stage cutaneous wound healing, and the second fraction promoting late stage wound healing, including epithelialization and rekeratinization. Merakris has successfully combined these with Dermacyte Matrix to demonstrate improved healing outcomes in cutaneous ulcers. Pursuant to recent US FDA guidance, Merakris has completed a pre-Investigational New Drug (IND) meeting with the FDA and is preparing an IND to further develop the use of this technology in combination with Dermacyte Matrix.

\section{Achievements}

\section{Allele}

Allele Biotechnology (CA, USA; www.allelebiotech.com) has announced a publication of a step-by-step protocol for manufacturers of iPSC and other types of stem cells with streamlined cleanroom practices for growing clinical grade cell products [5,6]. The paper also provides sources of cGMP-grade culture media, key reagents, equipment as well as the critical steps taken during splitting, stocking, and thawing of frozen iPSC stocks. This is the continuation of a series of protocol publications in the same journal, where Allele has provided procedures for mRNA reprogramming and generating iPSCs using cGMP materials. The mRNA reprogramming technology developed by Allele has proven to be highly efficient, consistent and reproducible. It allows generation of iPSCs that better recapitulate the molecular signatures of human embryonic stem cells and with faster kinetics than other methods. Differentiation potential of iPSC lines generated using published protocols by Allele have been tested toward more than a dozen different cell types successfully by several teams in the industry with minimal line-to-line variations. The company stated that this fully tested protocol for iPSC culture has been optimized for efficient iPSC proliferation and stemness. It allows scalable manufacturing operations that are fully compliant with cGMP standards; it can be adopted by any cGMP facility, providing a valuable platform to generate iPSCs for clinical applications. Moreover, this platform is designed to allow an easy transition to closed cell culture systems toward automation, which facilitates scalability and controllability and reduces operator-introduced variability, a direction of development Allele is actively engaged in.

\section{Auxolytic}

Auxolytic (UK; www.auxolytic.com) in collaboration with researchers at Stanford University School of Medicine (CA, USA; www.stanford.edu), demonstrated that it has developed a novel safety "off" switch for various types of cell therapies, potentially enabling a physician to mitigate serious side effects when they are observed $[7,8]$. The switch works by knocking out a uridine monophosphate synthase (UMPS), which is the gene that naturally synthesizes the nutrient uridine. The UMPS-edited cells are dependent on the administration of external uridine for their proliferation, enabling the control of their growth by modulating uridine supply. This approach was studied both in vitro and in vivo after transplantation in xenograft models. In xenograft models, researchers tested both a cell therapy and pluripotent stem cells that had been edited to require uridine. The data demonstrate that when uridine administration was halted, the cells were inactive and unable to proliferate within 1 week. The nature of the UMPS pathway enables a manufacturing process which yields a $100 \%$ consistent cellular product dependent on external uridine for survival.

In practice, patients would take a specific nutrient concurrent with the cell therapy. If serious side effects were observed, the nutrient would be discontinued, thus depleting the cell therapy in the body and reducing or stopping the side effects. 


\section{Clinical trials \\ Pluripotent stem cells \\ Cynata}

Cynata Therapeutics (Australia; www.cynata.com), a clinical stage biotechnology company specializing in cell therapeutics, has announced positive results from the two-year follow-up of patients enrolled in the Phase I clinical trial of CYP-001, Cynata's lead iPSC-derived Cymerus ${ }^{T M}$ mesenchymal stem cell (MSC) product [9]. The Phase I trial, which was the world's first clinical trial of an allogeneic iPSC-derived MSC product, evaluated the safety and efficacy of CYP-001 for the treatment of patients with steroid-resistant acute graft-versus-host disease (GvHD). The Phase I clinical trial enrolled 15 patients with steroid-resistant acute GvHD (https:/clinicaltrials.gov; ID: NCT02923375). The primary evaluation at Day 100 revealed highly promising safety and efficacy results, as announced on 18 December 2018. By Day 100, the complete response and overall response rates were 53 and $87 \%$, respectively, and overall survival (OS) was at least $87 \%$. Participants who completed the primary evaluation period then continued to a follow-up period for up to 2 years after the initial dose, which has now been completed. The OS rate after 2 years was 60\% (9/15 patients), which compares favorably with previously published outcomes. An OS rate of just $17 \%$ after 2 years has been reported in patients with steroid-resistant acute GvHD who received standard of care treatment. Furthermore, a recently published review article summarized numerous studies with bone marrow and adipose tissue derived MSCs in patients with steroid-resistant acute GvHD. Six of the studies reviewed reported two-year survival in MSC-treated patients, which ranged from 16.6 to $40 \%$. 2 Similarly, in another recently published Phase III trial of an investigational drug (ruxolitinib) in patients with steroid-resistant acute GvHD, OS after 12 months was 38\%, while there were insufficient surviving patients remaining on study to calculate the survival rate at later timepoints. The results of Cynata's trial illustrate the significant potential of CYP-001 as a new treatment option for GvHD. Study close-out procedures are now underway, which will include the preparation of a formal addendum to the clinical study report. Cynata's GvHD license partner, FUJIFILM (Japan; www.fujifilm.com), is responsible for the further development of CYP-001, with planning for a Phase II clinical trial advancing.

\section{Regulations, approvals, acquisitions... Green light Celyad}

Celyad Oncology (Belgium; www.celyad.com), a clinical-stage biotechnology company focused on the discovery and development of CAR T cell therapies for cancer, has announced that the company's IND application for CYAD-211, the company's first-in-class shRNA-based allogeneic CAR T candidate and second nongene edited offthe-shelf program, is in effect with the FDA [10]. The company's lead allogeneic candidate from its next-generation CYAD-200 series, CYAD-211 targets B-cell maturation antigen for the treatment of relapsed/refractory multiple myeloma (r/r MM). CYAD-211 is engineered to co-express a B-cell maturation antigen-targeting CAR and a single shRNA, which interferes with the expression of the CD3 component of the T-cell receptor complex.

Celyad Oncology is advancing a pipeline of proprietary, nongene edited allogeneic CAR T candidates from its CYAD-200 series, which is underpinned by its shRNA technology platform. The shRNA platform coupled with the company's all-in-one vector approach provides flexibility, versatility and efficiency to the design of novel, off-the-shelf CAR T candidates through a single step engineering process. Next-generation candidates exploring the breadth and depth of the Celyad Oncology shRNA platform are currently under preclinical development. Combining shRNA knockdown with additional functional components in a single CAR T construct may also offer therapeutic optionality to the nongene edited allogeneic CYAD-200 series of product candidates. In 2018, Celyad Oncology signed an exclusive agreement with Horizon Discovery Group for the use of its SMARTvector shRNA technology for the development of allogeneic CAR T therapies.

\section{Fate therapeutics}

Fate Therapeutics (CA, USA; https://fatetherapeutics.com) has announced that the FDA has cleared the Company's IND application for FT819, an off-the-shelf allogeneic CAR T-cell therapy targeting CD19 ${ }^{+}$malignancies [11]. FT819 is the first-ever CAR T-cell therapy derived from a clonal master iPSC line and is engineered with several first-of-kind features designed to improve the safety and efficacy of CAR T-cell therapy. The Company plans to initiate clinical investigation of FT819 for the treatment of patients with relapsed/refractory B-cell malignancies, 
including chronic lymphocytic leukemia (CLL), acute lymphoblastic leukemia (ALL) and non-Hodgkin lymphoma (NHL).

The Company's proprietary iPSC product platform enables mass production of off-the-shelf, engineered, homogeneous cell products that can be administered with multiple doses to deliver more effective pharmacologic activity, including in combination with cycles of other cancer treatments. Human iPSCs possess the unique dual properties of unlimited self-renewal and differentiation potential into all cell types of the body. The Company's first-of-kind approach involves engineering human iPSCs in a one-time genetic modification event and selecting a single engineered iPSC for maintenance as a clonal master iPSC line. Analogous to master cell lines used to manufacture biopharmaceutical drug products such as monoclonal antibodies, clonal master iPSC lines are a renewable source for manufacturing cell therapy products which are well-defined and uniform in composition, can be mass produced at significant scale in a cost-effective manner and can be delivered off-the-shelf for patient treatment. As a result, the Company's platform is uniquely capable of overcoming numerous limitations associated with the production of cell therapies using patient- or donor-sourced cells, which is logistically complex and expensive and is subject to batch-to-batch and cell-to-cell variability that can affect clinical safety and efficacy. Fate Therapeutics' iPSC product platform is supported by an intellectual property portfolio of over 300 issued patents and 150 pending patent applications.

The multicenter Phase I clinical trial of FT819 is designed to determine the maximum tolerated dose of FT819 and assess its safety and clinical activity in up to 297 adult patients across three types of B-cell malignancies (CLL, ALL and NHL). Each indication will enroll independently and evaluate three dose-escalating treatment regimens: Regimen A as a single dose of FT819; Regimen B as a single dose of FT819 with IL-2 cytokine support and Regimen C as three fractionated doses of FT819. For each indication and regimen, dose-expansion cohorts of up to 15 patients may be enrolled to further evaluate the clinical activity of FT819.

The Company's proprietary induced pluripotent stem cell iPSC product platform enables mass production of off-the-shelf, engineered, homogeneous cell products that can be administered with multiple doses to deliver more effective pharmacologic activity, including in combination with cycles of other cancer treatments. Human iPSCs possess the unique dual properties of unlimited self-renewal and differentiation potential into all cell types of the body. The Company's first-of-kind approach involves engineering human iPSCs in a one-time genetic modification event and selecting a single engineered iPSC for maintenance as a clonal master iPSC line. Analogous to master cell lines used to manufacture biopharmaceutical drug products such as monoclonal antibodies, clonal master iPSC lines are a renewable source for manufacturing cell therapy products which are well-defined and uniform in composition, can be mass produced at significant scale in a cost-effective manner and can be delivered off-the-shelf for patient treatment. As a result, the Company's platform is uniquely capable of overcoming numerous limitations associated with the production of cell therapies using patient- or donor-sourced cells, which is logistically complex and expensive and is subject to batch-to-batch and cell-to-cell variability that can affect clinical safety and efficacy. Fate Therapeutics' iPSC product platform is supported by an intellectual property portfolio of over 300 issued patents and 150 pending patent applications.

\section{Kite}

Kite (CA, USA; www.kitepharma.com), a Gilead Company (CA, USA; www.gilead.com), has announced that the FDA has granted accelerated approval to Tecartus ${ }^{T M}$ (brexucabtagene autoleucel, formerly KTE-X19), the first and only approved CAR T-cell therapy for the treatment of adult patients with relapsed or refractory mantle cell lymphoma (MCL). MCL is a rare form of NHL that arises from cells originating in the 'mantle zone' of the lymph node and predominantly affects men over the age of 60 [12]. MCL is highly aggressive following relapse, with many patients progressing following therapy. The approval of this one-time therapy follows a priority review and FDA Breakthrough Therapy Designation and is based on results of ZUMA-2, a single-arm, open-label study in which $87 \%$ of patients responded to a single infusion of Tecartus, including $62 \%$ of patients achieving a complete response. Among patients evaluable for safety, 18\% experienced Grade 3 or higher cytokine release syndrome and $37 \%$ experienced Grade 3 or higher neurologic toxicities. Tecartus is an autologous, anti-CD19 CAR T-cell therapy. Tecartus uses the XLPTM manufacturing process that includes T-cell enrichment, a necessary step in certain B-cell malignancies in which circulating lymphoblasts are a common feature. In addition to MCL, Tecartus is also currently in Phase I/II trials in ALL and CLL. The use of Tecartus in ALL and CLL is investigational and its safety and efficacy have not been established in these cancer types. 


\section{Orchard}

Orchard Therapeutics (UK \& MA, USA; www.orchard-tx.com), a global gene therapy company, has received both orphan drug designation and rare pediatric disease designation from the FDA for OTL-203, an ex vivo autologous hematopoietic stem cell (HSC) gene therapy in development for the treatment of mucopolysaccharidosis type I [13]. Orchard was granted an exclusive worldwide license to intellectual property rights to research, develop, manufacture and commercialize the gene therapy program for the treatment of mucopolysaccharidosis type I developed by the San Raffaele Telethon Institute for Gene Therapy (Italy; https://research.hsr.it/en/institutes/san-raffaele-telethoninstitute-for-gene-therapy.html).

\section{Talaris}

Talaris Therapeutics (MA, USA; www.talaristx.com), a privately held biotechnology company developing cell therapies, has announced that the FDA has approved the company's IND application for the evaluation of Talaris' novel cell therapy FCR001 in the treatment of diffuse systemic sclerosis, a severe form of the rare autoimmune disease scleroderma [14]. Approval of this IND allows Talaris to initiate a Phase I/IIa trial at multiple sites across the US. FCR001 is a cryopreserved allogeneic stem cell therapy derived from mobilized peripheral blood of the kidney donor that is delivered as a single dose in kidney transplant recipients who received a nonmyeloablative conditioning regimen. FCR001 contains the donor's CD $34^{+}$cells, facilitating cells and $\alpha \beta$ T-cells. This therapy induces or restores patients' immune tolerance by establishing stable donor chimerism in the transplant recipient without the need for life-long immunosuppression. FCR001 has received both Orphan Drug Designation and Regenerative Medicine Advanced Therapy designation from the FDA. A Phase III trial of FCR001 with 120 in living donor kidney transplant recipients, FREEDOM-1, is now enrolling patients; more information can be found at: http://freedom1study.com/ as well as www.clinicaltrials.gov, ID: NCT03995901.

\section{Name change \\ NanoSurface Biomedical into CuriBio}

NanoSurface Biomedical (WA, USA), a developer of human stem cell-based biotechnology products, has announced a comprehensive rebrand, including a change of the company's name to Curi Bio (www.curibio.com) [15]. To facilitate rapid growth of operational and $\mathrm{R} \& \mathrm{D}$ activities, including additional product and service offerings for pharma and biotech customers, Curi Bio has relocated its corporate headquarters to a new 5000 sqft biosafety level 2 laboratory and office space. The decision to take on a new name - Curi Bio - was driven by a strategic expansion of the core technology platforms to better serve our clients in the pharmaceutical industry. Curi Bio's suite of human stem cell-based products and services enable scientists to build mature and predictive human iPSC-derived tissues, with a focus on cardiac, musculoskeletal and neuromuscular models for discovery, safety testing and efficacy testing of new therapeutics. By offering drug developers an integrated platform comprising highly predictive human iPSC-derived cells and tissues and clinically relevant data, Curi Bio aims to dramatically accelerate the discovery and development of safer, more effective medicines.

\section{Acquisition \\ CuriBio \& Dana Solutions}

Curi Bio (WA, USA; www.curibio.com) has acquired Dana Solutions (CA, USA; www.danasolutionsllc.com), a pioneer in the application of artificial intelligence and machine learning to in vitro cell-based assays [16]. Through the acquisition, Curi Bio will gain access to Dana's artificial intelligence/machine learning-based platforms including PhenoLearn $^{T M}$, a deep learning platform for modeling cell and tissue phenotypes; Pulse ${ }^{T M}$, an automated platform for contractility analysis of beating cardiomyocytes and PhenoTox ${ }^{\mathrm{TM}}$, a patented deep learning platform for predictive safety pharmacology. Through a collaboration with the Health and Environmental Sciences Institute (HESI), Dana has previously received funding from the FDA to validate PhenoTox for the predictive assessment of drug-induced structural cardiac toxicity. Curi Bio and Dana will continue the FDA project following the acquisition. Dana's SaaS platforms will continue to operate until the expiration of existing customer contracts. By integrating Curi Bio's existing portfolio of human iPSC technology products with Dana's PhenoLearn and PhenoTox deep learning platforms, Curi Bio aims to help pharma customers bring safer, more effective medicines to market in less time, at lower cost. The detailed terms of the transaction were not disclosed. 


\section{Capital market \& finances Allovir}

AlloVir (MA, USA; www.allovir.com) was able with pricing its offering of 16.25 million shares at US\$17 apiece, to sell more shares than it planned, boosting the size of the stock offering from the initial target of 14.75 million shares and raising about US\$276.3 million to pull its cell therapies off the shelf and run multiple clinical trials testing them in stem cell and organ transplant patients [17]. AlloVir shares opened at US\$20 apiece, up $17.6 \%$ from the initial public offering (IPO) price.

AlloVir is developing cell therapies intended to treat and prevent viral infections in transplant patients. These patients have weak immune systems that make them particularly susceptible to infection. The standard of care includes antiviral drugs. But sometimes these drugs are not enough to stop viruses and these treatments can damage the kidneys. Instead of taking viruses head on, AlloVir's cell therapies are intended to restore the ability of a patient's immune system to tackle them.

The AlloVir cell therapies are called virus-specific T cells (VST). The process for making a VST therapy is similar to the way that CAR-T cancer treatments are produced. Immune cells are removed from the body and multiplied in a lab. But unlike CAR-T therapies, AlloVir's process does not engineer the cells in any way. The AlloVir cell therapies also do not start with a patient's own immune cells. The allogeneic approach uses the T cells of healthy people who have already been exposed to viruses. That exposure is expected to be enough get these cells to go after these viruses after the therapy has been infused into a transplant patient. Once produced, AlloVir's therapies can be stored and pulled 'off the shelf' for use as needed.

The most advanced AlloVir product candidate, Viralym-M, is a VST that targets five viruses: BK virus, cytomegalovirus, adenovirus, Epstein-Barr virus, and human herpesvirus 6 . The company is testing the cell therapy as a treatment for both stem cell transplant patients and organ transplant patients.

According to the prospectus, AlloVir plans to use the IPO cash to advance Viralym-M to a Phase III study testing it against hemorrhagic cystitis, inflammation of the bladder that is a virus-associated complication faced by stem cell transplant patients. The drug will also be tested in separate late-stage studies in cytomegalovirus and adenovirus. About US $\$ 98$ million is planned for those Phase III tests. Another US $\$ 83$ million is earmarked for Phase II tests of Viralym-M. Those tests will cover the prevention of multiple viral infections in stem cell transplant patients, treating BK virus infection in kidney transplant patients, and treating cytomegalovirus infections in solid organ transplant patients. The IPO cash will also support two respiratory virus programs. ALVR106 is being developed to treat respiratory syncytial virus, influenza, parainfluenza virus and human metapneumovirus. That VST is expected to start Phase I tests in the second half of this year. The other respiratory virus program, ALVR109, is in development as a treatment for infection by the novel SARS-CoV-2. The company plans to advance that program to Phase I/II testing.

\section{Lineage Cell Therapeutics}

Lineage Cell Therapeutics (CA, USA; www.lineagecell.com), a clinical-stage biotechnology company developing novel cell therapies for unmet medical needs, has announced that as a result of continued clinical progress, the Israel Innovation Authority (IIA; Israel; https://innovationisrael.org.il/en/contentpage/israel-innovation-authority) has extended its previously awarded development grant in support of OpRegen ${ }^{\circledR}$, the Company's human embryonic stem cell-derived retinal pigment epithelium cell transplant therapy in development for the treatment of dry age-related macular degeneration [18]. The research \& development grant of up to 9 million Israeli New Shekels (approximately US $\$ 2.5$ million) was awarded in 2019 by the IIA and has been extended for use through June 2021 based on promising clinical data from the ongoing Phase I/IIa clinical study of OpRegen. The IIA has provided annual grants totaling approximately US $\$ 16$ million for the development of the OpRegen program so far.

Vor

Vor Biopharma (MA, USA; www.vorbiopharma.com), an oncology company pioneering engineered hematopoietic stem cells for the treatment of cancer, has raised US $\$ 110$ million in a Series B financing [19]. Proceeds will advance the company's lead candidate VOR33 into clinical trials, deepen its portfolio and accelerate the validation of additional targets for its scientific platform, which is designed to remove redundant proteins so that transplanted stem cells become invisible to targeted therapies while leaving diseased cells vulnerable. Vor's treatment strategy 
has the potential to bring a revolutionary treatment paradigm for patients with acute myeloid leukemia and other hematologic malignancies.

Vor's lead product candidate, VOR33, consists of engineered hematopoietic stem cells that lack the protein CD33. Once these cells are transplanted into a cancer patient, Vor believes that CD33 will become a far more cancer-specific target, potentially avoiding toxicity to the normal blood and bone marrow associated with CD33targeted therapies. Vor aims to improve the therapeutic window and effectiveness of CD33-targeted therapies, thereby potentially broadening the clinical benefit to patients suffering from acute myeloid leukemia.

\section{Financial \& competing interests disclosure}

The author Dusko llic has received an honorarium from Future Science Group for the contribution of this work. The author has no other relevant affiliations or financial involvement with any organization or entity with a financial interest in or financial conflict with the subject matter or materials discussed in the manuscript apart from those disclosed.

No writing assistance was utilized in the production of this manuscript.

\section{References}

1. Fate Therapeutics. Fate Therapeutics announces exclusive license agreement with Baylor College of Medicine for rejection-resistant iPSC-derived cellular therapies (2020).

https://ir.fatetherapeutics.com/news-releases/news-release-details/fate-therapeutics-announces-exclusive-license-agreement-baylor

2. Mo F, Watanabe N, McKenna MK et al. Engineered off-the-shelf therapeutic T cells resist host immune rejection. Nat. Biotechnol. doi:10.1038/s41587-020-0601-5 (2020) (Epub ahead of print).

3. QurAlis. QurAlis announces agreement to strengthen ALS Pipeline (2020). https://quralis.com/quralis-announces-agreement-to-strengthen-als-pipeline/

4. BusinessWire. Merakris Therapeutics, LLC announces the commercial launch of Dermacyte ${ }^{\circledR}$ Matrix and CMS assignment of an HCPCS code for use as a skin graft substitute (2020). www.businesswire.com/news/home/20200709005591/en/Merakris-Therapeutics-LLC-Announces-Commercial-Launch-Dermacyte

5. Allele Biotechnologies. Allele discloses how its highly efficient iPSC culturing is conducted under cGMP (2020). https://www.allelebiotech.com/news

6. Rivera T, Zhao Y, Ni Y et al. Human-induced pluripotent stem cell culture methods under cGMP conditions. Curr. Protoc. Stem Cell Biol. 54(1), e117 (2020).

7. Auxolytic. Foundational Research published in Nature Biotechnology showcases auxolytic's approach to developing nutrient-based cell therapy control switches (2020). www.auxolytic.com/news/foundational-research-published-in-nature-biotechnology-showcases-auxol ytics-approach-to-developing-nutrient-based-cell-therapy-control-switches

8. Wiebking V, Patterson JO, Martin R et al. Metabolic engineering generates a transgene-free safety switch for cell therapy. Nat. Biotechnol. doi:10.1038/s41587-020-0580-6 (2020) (Epub ahead of print).

9. Cynata Therapeutics. Cynata completes Phase $1 \mathrm{GvHD}$ clinical trial follow-up with positive results (2020). http://files.cynata.com/592/200730PositiveResultsf romGvHDTrialFollow-up.pdf

10. Celyad Oncology. Celyad Oncology announces FDA clearance of IND application for CYAD-211, first shRNA-based, non-gene edited allogeneic CAR T therapy (2020). https://celyad.com/2020/07/14/celyad-oncology-announces-fda-clearance-of-ind-application-for-c yad-211-first-shrna-based-non-gene-edited-allogeneic-car-t-therapy/

11. Fate Therapeutics. Fate Therapeutics announces FDA clearance of IND application for first-ever iPSC-derived CAR T-cell therapy (2020).

https://ir.fatetherapeutics.com/news-releases/news-release-details/fate-therapeutics-announces-fda-clearance-ind-application-first

12. Kite Pharma. US FDA approves Kite's Tecartus ${ }^{\top M}$, the first and only CAR $T$ treatment for relapsed or refractory mantle cell lymphoma (2020). www.kitepharma.com/news/press-releases/2020/7/us-fda-approves-kites-tecartus-the-first-and-only-car-t-treatmen t-for-relapsed-or-refractory-mantle-cell-lymphoma

13. Orchard Therapeutics. Orchard Therapeutics announces Orphan Drug and Rare Pediatric Disease Designations for OTL-203 for the treatment of MPS-I (2020). https://ir.orchard-tx.com/node/7626/pdf

14. Talaris Therapeutics. FDA approves Talaris Therapeutics' IND for its allogeneic cell therapy FCR001 to be evaluated in patients with a severe form of scleroderma (2020). http://talaristx.com/2020/07/fda-approves-talaris-therapeutics-ind-for-its-allogeneic-cell-therapy- $\mathrm{fc}$ r001-to-be-evaluated-in-patients-with-a-severe-form-of-scleroderma/

15. Curi Bio. NanoSurface Biomedical is now Curi Bio (2020). www.curibio.com/news/nanosurface-biomedical-is-now-curi-bio

16. Dana Solution. Curi Bio acquires Dana Solutions (2020). www.curibio.com/news/curi-bio-acquires-dana-solutions

17. Vinluan F. Xconomy. AlloVir’s IPO raises $\$ 276 \mathrm{M}$ to test cell therapies in transplant patients (2020).

https://xconomy.com/boston/2020/07/30/allovirs-ipo-raises-276m-to-test-cell-therapies-in-transplant-patients/ 


\section{Industry News Ilic \& Liovic}

18. Lineage Cell Therapeutics. Lineage Cell Therapeutics announces extension of OpRegen ${ }^{\circledR}$ development grant from Israel Innovation Authority (2020). https://investor.lineagecell.com/node/20571/pdf

19. Vor Biopharma. Vor Biopharma closes $\$ 110$ million series B financing (2020). www.vorbiopharma.com/vor-biopharma-closes-110-million-series-b-financing/ 